\title{
Medición Simultánea Indirecta de la Presión Arterial en Lactantes Mediante Tres Métodos.
}

\author{
Dr. Sergio Scroggie A. ${ }^{3}$, Dra. Estela Gallardo G. ${ }^{2}$, Dra. Patricia Montenegro H. ${ }^{3}$, \\ Dra. Patricia Bondi H. ${ }^{4}$, Enf. Marlene Alfaro G. ${ }_{4}^{2}$, Enf. Patricia López F, \\ Enf. Silvia Guajardo N.
}

\section{Indirect Measurement of Arterial Blood Pressure in Children Using Three Simultaneous Methods}

\begin{abstract}
Arterial blood pressure was measured in 212 children 2, 3,12 and 36 months of age using 3 different techniques: Doppler, Flush and auscultation of Humeral and Tibial Posterior arteries.

In infants aged 2 and 3 months, pressure in Humeral Artery was higher than in the Tibial Posterior Artery. The figures are statistically significant. In children of one year of age pressure of Tibial Posterior Artery was higher than in Humeral Artery, difference which increases in measurements taken in 3 years olds.

The Transcutaneous Doppler Model 801-B only measures systolic pressure and gires different results as compared with the auscultatory method in Humeral Artery in 2 and 3 months olds infants. These differences disappear in children 1 and 3 years of age.
\end{abstract}

Anteriormente a la década presente se informaron muy pocos casos de hipertensión neonatal; sin embargo, en afros recientes se han registrado una cantidad de casos que sugiere, tal vez, de que la hipertensión en el recién nacido está en aumento. Existe consenso entre los investigadores de este tema de que esto se debe más bien, al uso corriente del Doppler Transcutáneo y de la cateterización intrarteriall en los Servicios de Recién Nacidos.

Los diversos factores que influyen en la presión del lactante menor, junto con el tiempo que se invierte en tomarla por una parte; y los errores de los métodos indirectos, que aunque incruentos, no dan seguridad al pediatra por otra, hace que este evite la decisión de cometer un error grave al diagnosticar una hipertensión arterial sin ésta existir; aunque creemos que el no detectar una real hipertensión es un error aún más grave.

Es por esta razón que cada día aumenta el convencimiento de incorporar al examen pediátrico la medición de la presión arterial tal como el pulso, peso y talla. Considerando esta necesidad, surge la importancia de buscar la certeza y precisión en la medición indirecta.

Moss y Adams (1962) usando la têcnica del "Flush" sobre muñeca y tobillo ien RN? encontraron diferencias de presión entre el brazo y la pierna, siendo en la primera superior a la segunda;

\footnotetext{
1 Presidente Liga Chilena de la Lactancia Materna

2 Consultorio Carlos Avendaño

${ }^{3}$ Consultorio Crecimiento y Desarrollo

4 Consultorio La Pincoya
}

contrariamente a lo que ocurre en el lactante de 1 año $y$ en el adulto, en quienes la presión de las piernas o pie es superior a la de los brazos.

Swiet, Peto y Schinebourne al medir con Doppler la presión de la arteria humeral y tibial posterior en Recién Nacidos encontraron una mayor presión en el brazo, sosteniendo que el itsmo aórtico estrecho a esta edad permite este fenómeno. Sin embargo, Nadas no concuerda con este hecho e insiste que la presión de las piernas es superior a la de los brazos debido a la suma de la onda anterógrada del pulso y deduce que la inversión en fayor de los brazos es patológica.

El propósito de este estudio es el de establecer de que hay cambios circulatorios del lactante menor al mayor y que estos tienen relación con la adaptación del feto a la vida extrauterina primero, luego con el mayor crecimiento de las piernas y posteriormente con la estación bípeda y marcha; específicamente cumple con el objetivo de comparar los métodos indirectos de medición de ia presión arterial en el lactante menor.

\section{MATERIAL Y METODO}

Durante los meses de Julio, Agosto y Septiembre (1981) se procedió a medir la presión arterial de 212 niños (106 de 2 meses, 66 de 3 meses, 25 de 1 año y 21 de 3 años) tomados de control sano de 3 Consultorios Infantiles del Gran Santiago.

Los requisitos de selección fueron los siguientes: Peso de nacimiento entre 2.600 y $4000 \mathrm{~g}$.; sin patologia perinatal ni actual. Previo a las medicio- 
nes se exigieron las siguientes condiciones: no haber ingerido medicamentos 4 ó 5 días antes; no haber recibido alimentos por lo menos 30 minutos antes del examen. Durante el examen se aceptaron sólo las presiones tomadas cuando el niño estaba tranquilo, con pulso inferior a 150 y se descartó a los que hubieran presentado vaciamiento vesical y/o intestinal durante la toma. La posición de los niños durante el examen fue siempre decúbitosupino.

Se usaron tres técnicas de medición de presión arterial: Doppler, Flush y Auscultatoria, para to cual se usaron: un Doppler Transcutáneo modelo 801-B de Park Electronic, un esfigmomanómetro Tycos con un manguito tipo B de $25 \mathrm{~cm} \mathrm{x} 5 \mathrm{~cm}$ especial para niños de $0-3$ meses y tipo $C$ de 29 cm $x 7 \mathrm{~cm}$ para 4 meses a tres afros de Kaniya Tsusan Kaisha.

El procedimiento comenzó con la colocación del brazalete correspondiente a la edad del nino en el brazo derecho (arteria humeral) y luego en la pierna a $1 \mathrm{~cm}$ del tobillo (arteria tibial posterior), realizando primero la Técnica Doppler y luego el método del Fush, contabilizando el pulso central y periférico.

La presión del manguito se bajó a razón de 2 a $3 \mathrm{~mm} \mathrm{Hg}$ por segundo, tanto en el Flush como en el Doppler. Cada 50 mediciones se conectó el manguito a un manómetro de $\mathrm{Hg}$ para control de calibración. Se mantuvo el esfigmomanómetro a la altura del corazón.

El registro fue hecho por dos personas en forma simultánea, conectando un par de audífonos al sistema y una tercera persona que anotaba los registros en el Doppler.

Con la técrica Doppler se tomó 2 registros a cada niño con 3 minutos de intervalo, cuyos resultados se promediaron.

\section{RESULTADOS:}

Tabla 1.

Presiones en arterias Humeral y Tíbial posterior a las 2 y 3 meses de edad con Técnicas Dopple y Flush ( $\overline{\mathrm{X}}$ y $\mathrm{S})$

2 Meses (106 nitños)

3 Mescs ( 66 niños)

HUMERAL

TIBIAL $P$.

HUMERAL

TIBIAL P.

\begin{tabular}{|c|c|c|c|c|c|c|c|c|c|c|c|c|c|c|c|}
\hline & $\overline{\mathrm{x}}$ & & $\mathrm{s}$ & & $\overrightarrow{\mathrm{x}}$ & & $\mathrm{s}$ & & $\overline{\mathrm{x}}$ & & $\mathbf{s}$ & & $\overrightarrow{\mathrm{x}}$ & & $s$ \\
\hline Doppler & 113.66 & & \pm 13.91 & & 91.13 & & \pm 13.06 & & 118.07 & & \pm 16.47 & & 98.18 & & \pm 13.99 \\
\hline \multirow[t]{3}{*}{ Flush } & 68.72 & & \pm 9.48 & & 53.66 & & \pm 8.16 & & 68.71 & & \pm 10.27 & & 55.19 & & \pm 10.22 \\
\hline & \multicolumn{3}{|c|}{ HLMERAL } & & \multicolumn{3}{|c|}{ TIBIAL P. } & & \multicolumn{3}{|c|}{ HUMERAL } & \multicolumn{4}{|c|}{ TIBIAL P. } \\
\hline & Máx. & & Min. & & Máx. & & Mín. & & Máx. & & Min. & & Máx. & & Mín. \\
\hline Dopplet & 140 & - & 80 & - & 120 & - & 70 & - & 150 & - & 85 & - & 140 & - & 75 \\
\hline Flush & 100 & - & 40 & - & 75 & - & 40 & - & 100 & - & 40 & - & 80 & - & 40 \\
\hline
\end{tabular}

$\overline{\mathrm{X}}$ : Promedio-S: Desviación estandard.

La Tabla 1 presenta los promedios y desviaciones standards de las presiones tomadas a los nifios de 2 y 3 meses de edad, humerales y tibiales posteriores, con las técnicas de Doppler y Flush.

Como se observa las presiones humerales con ambas técnicas, en los dos grupos de edades, son más altas que las presiones tibiales. Estas diferencias son estadísticamente significativas.

Sin embargo si se compara esta diferencia entre los dos grupos, se observa una diferencia ligeramente mayor a los 2 meses que a los tres meses entre presión humeral y tibial.

También el cuadro nos pernite ver cómo la presión tibial a los 3 meses, en ambas técricas va aumentando si se la compara con la presión tibial a los 2 meses.

Sin embargo, al año de edad las presiones tibiales son más altas que las humerales y continúan en aumento a los 3 años. Tabla 2 .

El cuadro expone, además, las presiones máximas y mínimas registradas en ambos grupos y con ambas técnicas.

Tabla 2.

Promedio de presiones humerales y tibiales posteriores a los 3 meses, 1 año y 3 años tomadas con la técnica Doppler

\begin{tabular}{|c|c|c|c|c|c|}
\hline \multicolumn{2}{|c|}{3 moses (66 niflos) } & \multicolumn{2}{|c|}{ ป aก็o (25 niñ os) } & \multicolumn{2}{|c|}{3 años $\{21$ niflos $\}$} \\
\hline Humetal & Tibjakes P. & Humeral & Tjbioles $\mathbf{P}$. & Hurneral & Tobiales $P$. \\
\hline $\overrightarrow{\bar{x}}$ & $\overline{\bar{x}}$ & $\overline{\bar{x}}$ & $\overline{\mathrm{x}}$ & $\overline{\mathbf{x}}$ & $\overline{\mathrm{x}}$ \\
\hline 118.07 & 98.18 & 105.8 & 110.8 & 106.57 & 124.33 \\
\hline
\end{tabular}

En efecto, en la Tabla 2 se aprecia que la presión tibial posterior es mayor que la humeral al 
año de edad, diferencia que se acentúa aún más a los tres años. Esto permite establecer que la arteria tibial posterior sufre cambios tensionales desde el nacimiento al affo de edad; ya sea por un estrechamiento del itsmo aórtico, adaptación de la circulación fetal a la extrauterina y mayor crecimiento de las piernas desde el nacimiento al año, lo que se acentúa con la estación bípeda o marcha.

Con el objeto de establecer comparaciones entre los métodos Doppler y auscultatorio, se emplearon simultáneamente estas dos técnicas en los nifos de 1 año y 3 afios. En la Tabla 3 se presentan los promedios y desviaciones standards de las presiones tomadas.

Tabla 3.

Comparación de presiones arteriales de nijos de 1 y 3 años obtenidas con técnicas de Doppler y auscultatoria ( $\overline{\mathrm{X}}$ y $\$$ )

\begin{tabular}{|c|c|c|c|c|c|}
\hline \multirow{2}{*}{\multicolumn{2}{|c|}{$\begin{array}{c}1 \text { Año (25 Casos) } \\
\text { Auscultatoria }\end{array}$}} & \multicolumn{4}{|c|}{1 Año (25 Casos) } \\
\hline & & \multicolumn{2}{|c|}{ Doppler Humeral } & \multicolumn{2}{|c|}{ Doppler Tíbial P. } \\
\hline$\overline{\mathrm{X}}$ & s & $\overline{\mathrm{x}}$ & $\mathrm{s}$ & $\overline{\mathrm{x}}$ & $\mathrm{s}$ \\
\hline 103.12 & \pm 9.03 & 105.8 & \pm 10.38 & 110.8 & \pm 13.04 \\
\hline \multicolumn{2}{|c|}{3 Años (21 Casos) } & \multicolumn{4}{|c|}{3 Años (21 Casos) } \\
\hline \multicolumn{2}{|c|}{ Auscultatoria } & \multicolumn{2}{|c|}{ Doppler Humeral } & \multicolumn{2}{|c|}{ Dopplex Tibial P } \\
\hline$\overline{\mathrm{X}}$ & $\mathrm{s}$ & $\overrightarrow{\mathrm{x}}$ & $s$ & $\overline{\mathrm{x}}$ & s \\
\hline 105.33 & \pm 6.14 & 106.57 & \pm 5.79 & 124.33 & \pm 12.68 \\
\hline
\end{tabular}

Se aprecia en la tabla que los promedios obtenidos por la técrica auscultatoria en las edades de 1 y 3 años tienden a igualarse con los del Doppler aplicado en el brazo, no se encontraron diferencias estadisticamente significativas entre ambos.

Este fenómeno ha sido registrado por numerosos autores que pretenden significar que el Doppler mide iguales presiones que la auscultatoria y que la presión intraarterial tomada con electrodos.

Tabla. 4.

Ptesiones tomadas a niños de 2 y 3 meses con las técnicas Doppler y auscultatoria ( $\overline{\mathrm{X}}$ y $\mathrm{S}$ )

2 Meses (13 Casos) 2 Meses (13 Casos)

Auscultatoria Humeral Doppler Humeral Doppler Tibial P.

$\begin{array}{cccccc}\bar{X} & S & \bar{X} & S & \bar{X} & S \\ 107.69 & 7.25 & 120.38 & 5.94 & 93.07 & 13,77\end{array}$

3 Meses (19 Casos) 3 Meses (19 Casos)

Auscultatoria Humeral Doppler Humeral Dopplet Tjbial P.

\begin{tabular}{cccccc}
$\overline{\mathrm{X}}$ & $\mathrm{S}$ & $\overrightarrow{\mathrm{X}}$ & $\mathrm{S}$ & $\overline{\mathrm{X}}$ & $\mathrm{S}$ \\
110.26 & 11.36 & 118.31 & 12.36 & 94.73 & 12.96 \\
\hline
\end{tabular}

En la Tabla 4 se puede observar que a los 2 y 3 meses, las cifras de presión registradas con et Doppler transcutáneo resultan mayores que las obtenidas con el método auscultatorio sobre la arteria humeral. Sin embargo la presión sistólica tomada con el método auscultatorio siempre estuvo entre la presión Doppler humeral y Doppler tibial posterior.

Tabla 5.

Comparación de presiones tomadas con la técnica auscultatoria entre las edades:

2 mescs, 3 meses, 1 año, 3 años.

\begin{tabular}{lrrr}
\hline & \multicolumn{2}{c}{ Auscultatoria } & Pulso \\
EDADES & $\overline{\mathrm{X}}$ & $\mathrm{S}$ & $\overline{\mathrm{X}}$ \\
\hline 2 meses (13 casos) & 107.69 & 7.25 & 138 \\
\hline meses (19 casos) & 110.26 & 11.36 & \\
\hline 1 año (25 casos) & 103.12 & 9.03 & 109 \\
3 años (21 casos). & 105.33 & 6.14 & 103 \\
\hline
\end{tabular}

Otro resultado llamativo de este estudio es la diferencia encontrada entre la presión auscultatoria humeral en lactantes menores y nifios de 1 año y 3 años, ver Tabla 5 .

Sin embargo, considerando la edad y el peso creemos que en el lactante de 2 y 3 meses la presión auscultatoria tiene un alza relativa en relación al niño de 1 y 3 años. La taquicardia sinunal del lactante menor, los cambios circulatorios que se han señalado, al parecer influencian una relativa presión más alta en los brazos; la que va decreciendo con la edad en la medida que la presión de la tibial posterior va subiendo. Este resultado evidencia la utilidad de medir la presión en brazo y pierna simultáneamente con Doppler y si la presíbn está levemente aumentada en los brazos, tanto la sistólica como diastólica, se debe medir varias veces auscultatoriamente hasta $\operatorname{los} 6,7,8$ meses antes de efectuar el estudio completo de hipertensión.

\section{DISCUSION:}

El problema inicial que se presentó fue la elección de mango. Se eligió el "KTK Blood Pressure minicuff" de Kamiya Tsusan Kaisha de $23 \mathrm{~cm}$ de largo $\times 5 \mathrm{~cm}$ de ancho correspondiente a niños de 0-3 meses y que posee un sistema que permite observar el perimetro de la extremidad y si la goma cubre en su totalidad. Se.descartaron prematuros que deberian haber sido evaluados con mango más chico. En todos los ninos de 2 y 3 meses se usó el mismo mango. En los niños de 1 y 3 años las mediciones se hicieron con el mango tipo $\mathrm{C}$ de $29 \mathrm{~cm} \times 7 \mathrm{~cm}$ de la misma compañía. Moss y asociados recomiendan un mango de $5 \mathrm{~cm}$. Sullivan y Kosayashi no encontraron diferencias entre el brazo y ja pierna con mango de $4.5 \mathrm{~cm}$ con la técnica de Flush. Kirkland y Kirkland, 
usando mango de $6.3 \mathrm{~cm}$ encontraron una presión más alta en la arteria ploplítea que en la humeral con el Doppler Transcutáneo.

Como resultado de todo esto, la elección del mango apropiado, técnica y la arteria a medir dispersan y desorientan al pediatra con distintos resultados.

En este estudio se prefirió no cambiar de mango y utilizar la arteria tibial posterior que presenta además otras ventajas como comodidad, fäcil acceso y menor variación con el llanto, inquietud, etc.

Consideramos que la técnica del Flush es económica pero insegura ya que el grupo, después de medir 1000 presiones, aún tenj́a divergencias en la lectura del resuitado.

Por otra parte, los ruidos arteriales no se pueden oír siempre en lactantes pequeños y debe hacerlo una sola persona que comúnmente queda insegura y debe solicitar a otra su comprobación.

E1 Doppler se maneja con dos personas, to que facilita la certificación del primer sonido. Esta técnica no registra presión diastólica y da lecturas humerales más altas en lactantes menores.

Por otra parte, la presión sistólica auscultatoria siempre se encuentra entre la presión Doppler humeral y Doppler tibial posterior, nunca por encima o debajo.

A través de esta experiencia, recomendamos que, pesquisada una cifra alta de presión sistólica se hagan varias mediciones auscultatorias antes de formular el diagnóstico de hipertensión. Consideramos que el método Doppler es un instrumento útil y sencillo para detectar posibles pacientes hipertensos.

\section{AGRADECIMIENTOS:}

A los Directores de Consultorio, Dres.: Haydeé Sepúlveda de Bórquez (Consultorio Pincoya), Agust ín Cruz Melo (Consultorio Independencia), Paula Peláez (Consultorio Carlos Avendaño) y al Dr. Sergio González (Médjco Pediatra Unidad Salud Infantil del Ministerio de'Salud), por la cola boración que hizo posible la realización de este Trabajo.

\section{RESUMEN:}

Se midió la Presión Arterial en 212 niños de 2, 3,12 y 36 meses de edad de tres Consultorios de la Región Metropolitana con 3 técnicas diferentes: DOPPLER, FLUSH Y AUSCULTATORIA EN ARTERIA HUMERAL Y TIBIAL POSTERIOR.

En los niños de 2 y 3 meses de edad la presión en la Arteria Humeral fue mayor que la registrada en la Arteria Tibial Posterior. Las diferencias son. estadísticamente significativas. En los nifíos de I año de edad la presión en la Arteria Tibial Poste- rior fue mayor que en la Arteria Humeral lo que se acentúa en la medición hecha en niflos de 3 años de edad.

El Doppler Transcutáneo Modelo 801-B sólo mide Presión Sistólica y produce resultados diferentes al método con auscultatorio en lactantes de 2 y 3 meses sobre Arteria Humeral. Estas diferencias que disminuyen en niños de uno y tres haciéndose no significativa.

En los lactantes de 2 y 3 meses la presión sobre Arteria Humeral medida con Doppler Transcutáneo Modelo 801-B fue mayor que en los nifros de 1 y 3 años de edad.

\section{REFERENCIAS}

${ }^{1}$ Kirkland, M.D. and Kirkland J. M.D. et al.: Systotic blood pressure measurement in the newborn infant with the Transcutaneous Doppler Method. January 1972 The Journal of Pediatrics. Vol. 80, No I pp 52-56.

2 Swief M., Peto $J_{1}$ and Shinebournee E.A. et al.: Difference between upper and lower limb blood pressure in normal Neonates using Doppler Technique Archives of disease in Childhood, 1974 pp 49,734, 735.

${ }^{3}$ Mos Arthur, M.D. ef al.: Indirect Methods of Blood pressure measurement. Pediatric Clinics of North America. Vol. 25 No 1, February 1978, pp 3-25.

4 Adelman Raymond $D$. ef al: Neonatal Hypertension. Pediatric Clinics of North America. Vol. $25 \mathrm{~N}^{\circ} 1$, February 1978, pp 99-109.

s Goldring David M.D. et al.: Treatment of the Infant and Child with Coartation of the Aorta. Pediatric Clinics of North Ametica. Vol. 25 No 1, February 1978, pp 111.

6 Nados. et al: : Texto Cardiología Pediátrica. 1975.

${ }^{7}$ Berenson G.S., Foster, T.4. Frank G.C. et al.: Cardiovascular Disease risk factor variables at the preschool age, Unpublished report.

${ }^{8}$ Blumental S. Epps, R.P. Heavenrich R., et al.: Report of the Task force on blood pressure control in children. Pediatrics 59,$797 ; 1977$.

${ }^{9}$ Gyton A.C. Cowley A.W. Coleman, T.G. et al.: Hypertension, a disease of abnormal circulatory control. Chest, 65, 328, 1974 .

10 Hamilson M., Pickering G.W., Fraser-Roberts J.A, et al.: The aetiology of essential hypertension. I. The arterial pressure in the general population. Clin. Sci. 13: 11,1954 .

11 Johnson, A.L. Cornoni, J.C. Cassel, J.C. ef at.: Influence of race, sex and weight on blood pressure behaviour in young adults.

12 Moss, A.J. and Adams, F.H. Flush blood pressure and Intraarterial pressure: A comparison of Methods in infants, Am. J. dis. child. 107, 498; 1964.

13 Moss A.S. Liebling $W$, and Adams, F.H. The flush method for determining blood pressure in infants. Il normal values during the frrst year of life. Pediatrics $21: 950 ; 1958$.

14 Moss A.J., Duffie E.R., and Emmonoullides, G.C. Blood pressure and vasomotor reftexes in the Newtorn infants. Pediatrics 32, 175; 1963.

15 Ashworth A.M. and Neligan, G.A. changes in the systolic blood pressure of normal babies during the first twenty four hours of life, lancet 1 : $804 ; 1959$. 